\title{
Severe riboflavin deficiency induces alterations in the hepatic proteome of starter Pekin ducks
}

\author{
Jing Tang ${ }^{1,2}$, Maria A. Hegeman ${ }^{3}$, Jian $\mathrm{Hu}^{1}$, Ming Xie ${ }^{1}$, Wenbiao Shi, ${ }^{1,3}$, Yong Jiang ${ }^{1}$, Vincent de Boer ${ }^{3}$, \\ Yuming $\mathrm{GuO}^{2}$, Shuisheng $\mathrm{Hou}^{1}{ }^{*}$ and Jaap Keijer ${ }^{3}$ \\ ${ }^{1}$ Institute of Animal Sciences, Chinese Academy of Agricultural Sciences, Beijing 100193, People's Republic of China \\ ${ }^{2}$ College of Animal Science and Technology, China Agricultural University, Beijing 100193, People's Republic of China \\ ${ }^{3}$ Human and Animal Physiology, Wageningen University, 6700 AH Wageningen, the Netherlands
}

(Submitted 14 February 2017 - Final revision received 9 August 2017 - Accepted 7 September 2017)

\section{Abstract}

Suboptimal vitamin $\mathrm{B}_{2}$ status is encountered globally. Riboflavin deficiency depresses growth and results in a fatty liver. The underlying mechanisms remain to be established and an overview of molecular alterations is lacking. We investigated hepatic proteome changes induced by riboflavin deficiency to explain its effects on growth and hepatic lipid metabolism. In all, 360 1-d-old Pekin ducks were divided into three groups of 120 birds each, with twelve replicates and ten birds per replicate. For $21 \mathrm{~d}$, the ducks were fed ad libitum a control diet (CAL), a riboflavin-deficient diet (RD) or were pair-fed with the control diet to the mean daily intake of the RD group (CPF). When comparing RD with CAL and CPF, growth depression, liver enlargement, liver lipid accumulation and enhanced liver SFA (C6: 0, C12:0, C16:0, C18:0) were observed. In RD, thirty-two proteins were enhanced and thirty-one diminished ( $>1.5$-fold) compared with CAL and CPF. Selected proteins were confirmed by Western blotting. The diminished proteins are mainly involved in fatty acid $\beta$-oxidation and the mitochondrial electron transport chain (ETC), whereas the enhanced proteins are mainly involved in TAG and cholesterol biosynthesis. RD causes liver lipid accumulation and growth depression probably by impairing fatty acid $\beta$-oxidation and ETC. These findings contribute to our understanding of the mechanisms of liver lipid metabolic disorders due to RD.

Key words: Riboflavin deficiency: Lipid metabolism: Liver proteomics: $\beta$-Oxidation: Electron transport chain

Suboptimal riboflavin status occurs in children, elderly and women worldwide, particularly in regions with low intakes of dairy products and meat ${ }^{(1-6)}$. Riboflavin is an essential precursor for the biosynthesis of FMN and FAD. A number of flavindependent proteins that utilise FMN or/and FAD, so-called flavoproteins, participate in a range of redox reactions in, among others, the tricarboxylic acid (TCA) cycle, fatty acid $\beta$-oxidation, amino acid degradation and the electron transport chain (ETC) $)^{(1,7)}$. Owing to its involvement in primary metabolic pathways, riboflavin deficiency (RD) causes impaired growth, glossitis, anaemia and dermatitis in humans ${ }^{(8-10)}$. Also in animal studies, RD depresses growth and even results in death of rats $^{(11-15)}$, fish ${ }^{(16)}$ and ducks ${ }^{(17-19)}$. Disturbed lipid handling was shown to be a major consequence of RD in both humans and animals. Riboflavin intakes are inversely associated with trunk fat mass and total body fat mass in children ${ }^{(20)}$. In addition, RD was shown to provoke fatty livers in cats ${ }^{(21)}$ and rats ${ }^{(22)}$ and to alter the liver fatty acid composition of rats ${ }^{(12,22,23)}$. Several studies in rodents have shown that RD impairs hepatic mitochondrial $\beta$-oxidation of fatty acids by reducing the activities of the fatty acyl-CoA dehydrogenases ${ }^{(12,15,24-27)}$, which are flavin-dependent enzymes catalysing the rate-limiting, initial step of $\beta$-oxidation ${ }^{(28)}$.

While it is clear that RD affects growth and lipid metabolism, an experimental overview of underlying processes has not been presented and the extent of changes induced by RD have not been investigated at the protein level - the functional relevant integration level. Here, we used a proteomic approach in starter Pekin ducks, a well-established model of RD, to investigate the effects of RD on hepatic protein levels in relation to changes in hepatic lipid profile.

\footnotetext{
Abbreviations: ACAD9, acyl-CoA dehydrogenase family member 9; ACADM, medium-chain specific acyl-CoA dehydrogenase; ACADS, short-chain specific acyl-COA dehydrogenase; ACAT2, acetyl-COA acetyltransferase; APOB, apo B-100, CAL, fed ad libitum a control diet; CPF; pair-fed with the control diet to the mean daily intake of the riboflavin-deficient group; DLD, dihydrolipoyl dehydrogenase; ETC, electron transport chain; ETFDH, electron transfer flavoproteinubiquinone oxidoreductase; FXN, frataxin; HMGCS1, hydroxymethylglutaryl-CoA synthase; MDH1, malate dehydrogenase; MTTP, microsomal TAG transfer protein; NDUFA8, NADH dehydrogenase (ubiquinone) $1 \alpha$ subcomplex subunit 8; NDUFS1, NADH-ubiquinone oxidoreductase 75 kDa subunit; RD, riboflavindeficient diet; SCP2, non-specific lipid-transfer protein; TC, total cholesterol; TCA, tricarboxylic acid.
} 


\section{Methods}

\section{Animals and housing}

All procedures were approved by Animal Welfare Committee of Institute of Animal Sciences, Chinese Academy of Agricultural Sciences. A total of 360 1-d-old male white Pekin ducks (Anas platyrbynchos) were obtained from the Pekin duck breeding center (Chinese Academy of Agricultural Sciences) and randomly assigned to thirty-six raised plastic-floor pens with ten birds per pen. All ducks were divided into three experimental groups, each containing twelve replicates with ten birds per replicate. The ducks were either ad libitum fed with a control diet (CAL) or an RD diet, or pair-fed with a control diet based on the mean intake of the RD group (CPF). All ducks were fed their respective diets and had free access to water from the day of hatching to $21 \mathrm{~d}$ of age. During this period, the light was continuously on. The temperature was kept at $33^{\circ} \mathrm{C}$ from 1 to $3 \mathrm{~d}$ of age, after which it was gradually reduced to approximately $25^{\circ} \mathrm{C}$ until $14 \mathrm{~d}$ of age and kept at approximately $16-22^{\circ} \mathrm{C}$ for the remainder of the experiment.

\section{Diet}

The basal diet during the experimental period was riboflavindeficient containing $1.38 \mathrm{mg}$ of free riboflavin/ $\mathrm{kg}$ of diet (online Supplementary Table S1). The RD diet and CAL were produced from the basal diet, and were supplemented with, respectively, 0 and $10 \mathrm{mg}$ of crystalline riboflavin $/ \mathrm{kg}$ diet (purity, 99\%; Sigma-Aldrich). The riboflavin concentration for the CAL met the recommendations for starter ducks provided by the National Research Council ${ }^{(29)}$.

\section{Sampling}

To pair-feed the CPF group with a CAL, daily feed intake of the RD ducks was recorded to determine the mean intake. At $21 \mathrm{~d}$ of age, body weight and feed intake of each pen were measured. One duck from each pen was randomly selected and bled by cardiac puncture. Blood was collected into heparin-Nacontaining tubes, and centrifuged at $1520 \boldsymbol{g}$ for $10 \mathrm{~min}$ to obtain plasma, which was then stored at $-20^{\circ} \mathrm{C}$ until further analysis. Afterwards, these ducks were killed by $\mathrm{CO}_{2}$ inhalation and livers were dissected, weighed, snap-frozen in liquid $\mathrm{N}_{2}$ and stored at $-80^{\circ} \mathrm{C}$ until further analysis.

\section{Riboflavin, FMN and FAD content}

The free riboflavin content in feed, plasma and liver and the FMN and FAD content in liver were determined by reversedphase HPLC according to methods described previously ${ }^{(17,18)}$. Feed, liver and plasma samples were prepared before HPLC analysis according to the method described previously ${ }^{(30-32)}$. Peaks were identified and quantified using pure authentic standards of riboflavin, FMN and FAD (Sigma-Aldrich).

\section{Plasma parameters}

Plasma parameters, including glucose, lactate, uric acid, pyruvate, albumin, alanine transaminase (ALT), aspartate transaminase
(AST), TAG and total cholesterol (TC), were determined using commercial kits according to the manufacturer's instructions (Biosino Bio-Technology and Science Inc.).

\section{Liver lipids}

Total lipids were extracted by homogenising minced liver tissue samples in chloroform-methanol (2:1) according to ${ }^{(33)}$. The extracts were evaporated under a stream of nitrogen, weighed and resuspended in chloroform-methanol (2:1) containing $0.01 \%$ butyrated hydroxytoluene. Measurements of total lipids in liver were performed as described ${ }^{(34)}$. Aliquots were dried and resuspended in 1-butanol for analysis of TAG and TC using commercial kits according to the manufacturer's instructions (Biosino Bio-Technology and Science Inc.). Fatty acid methyl esters were prepared by acid-catalysed transmethylation of total lipids using boron trifluoride methanol according to the method described previously ${ }^{(35)}$. Fatty acid composition of liver was analysed in a gas chromatograph (Agilent 6890; Agilent Technologies) as described previously ${ }^{(36)}$. Peaks were identified by comparing retention times with those of the corresponding standards (Sigma-Aldrich).

\section{Liver proteomics}

To reduce the influence of individual variation, twelve individual liver samples per group were used to conduct the isobaric tags for relative and absolute quantification (iTRAQ) assays. A pooled sample in each group was created from an equal amount of protein from each of twelve samples. Proteins were extracted and digested as described ${ }^{(37)}$. Each digested sample was labelled with iTRAQ 8-plex reagents (AB Sciex) as follows, according to the manufacturer's instructions: tag 117 for RD, tag 118 for CAL and tag 119 for CPF. Labelled samples were mixed and fractionated into twenty fractions by HPLC (Ultimate 3000 BioRS; Dionex-Thermo Fisher Scientific Inc.) using a Durashell C18 $(5 \mu \mathrm{m}, 100 \AA, 4.6 \times 250 \mathrm{~mm})$ column. Liquid chromatography-electrospray ionisation-MS/MS analysis was carried out with a Triple TOF 5600 plus system (AB Sciex). The original MS/MS file data for identification and quantification were analysed against the database Uniprot_Neognathae_8825 using ProteinPilot Software version 4.0 (AB Sciex). Only unique peptides whose confidence intervals are more than $95 \%$ were contained in the iTRAQ labelling quantification and used for further analysis.

For analysis of the proteomic results, the relative expressions of identified proteins were based on the ratio of the reporter ions of the peptides between two of three groups (i.e. RD $v$. $\mathrm{CPF}, \mathrm{RD} v$. CAL, CPF $v$. CAL). Fold changes (FC) were used to determine whether identified proteins were enhanced (FC $>1.5$ ) or diminished $(\mathrm{FC}<-1.5)$ by $\mathrm{RD}$.

To enrich the differentially expressed proteins with respect to specific functional terms, the protein lists were analysed using the ClueGo software (http://www.ici.upmc.fr/cluego/) with the Gene Ontology database (release date: March 2016). The ontology selection on the base of biological processes and enrichment analysis was performed by the right-side hypergeometric statistic test and its probability value was corrected by the Bonferroni method ${ }^{(38)}$. 
A pathway enrichment analysis of the differentially expressed proteins ${ }^{(39)}$ was performed using ClueGo software and applying database from the Kyoto Encyclopedia of Genes and Genomes (KEGG) database (release date: February 2016).

\section{Western blot analyses}

Western blot analysis of the liver samples of ducks was conducted as described ${ }^{(40)}$. Primary antibodies $(1 \mu \mathrm{g} / \mathrm{ml})$ against short-chain specific acyl-CoA dehydrogenase (ACADS) (ab154823), medium-chain specific acyl-CoA dehydrogenase (ACADM) (ab92461) and acyl-CoA dehydrogenase family member 9 (ACAD9) (ab113917) were obtained from Abcam. Blots were stripped and re-probed with antibodies against $\beta$-actin (ACTB; HX1831; Huaxing-Bio) to demonstrate equal sample loading.

\section{Statistical analyses}

One-way ANOVA analysis followed by Tukey's multiple comparison was performed using SAS software (2003; SAS Institute). Data were expressed as mean values with their standard errors. A probability level of $P<0.05$ was considered to be statistically significant.

\section{Results}

\section{Mortality and growth performance}

The mortality of ducks was greater in RD than in CAL and CPF $(P<0.001$; online Supplementary Table S2). Growth performance of the ducks at $21 \mathrm{~d}$ of age was assessed by average daily weight gain (ADG), average daily feed intake (ADFI) and gain to feed ratio (G:F) (online Supplementary Table S2). ADG and ADFI declined in $\mathrm{RD}$ and $\mathrm{CPF}$ in comparison with $\mathrm{CAL}$ $(P<0.001)$. ADG was lower in RD than in CPF $(P<0.001)$, even though the ADFI was equal in these two groups. G:F was lower in RD than in CAL and CPF $(P<0.001)$. The relative liver weight was elevated in the RD group compared with CAL and CPF $(P<0 \cdot 001)$

\section{Riboflavin status}

Plasma riboflavin concentration decreased by $88 \%$ as a result of RD ( $P<0.001$; online Supplementary Fig. S1(A)). Liver riboflavin and FMN concentrations were reduced in RD compared with CAL and CPF $(P<0 \cdot 001$; online Supplementary Fig. S1(B)). Liver FAD concentration was decreased in both $\mathrm{RD}$ and $\mathrm{CPF}$ when compared with CAL $(P=0 \cdot 008$; online Supplementary Fig. S1(B)).

\section{Plasma parameters}

Plasma glucose and lactate did not differ among RD, CPF and CAL. Plasma uric acid, pyruvate, albumin, TAG and TC levels were elevated in RD compared with $\mathrm{CPF}$ and CAL $(P<0.05$; Table 1$)$. Plasma enzyme activities of ALT and AST were greater in RD than in CPF and CAL $(P<0.001$; Table 1).

\section{Liver total lipids, TAG, total cholesterol and fatty acid composition}

Liver total lipids, TAG and TC content were elevated in RD when compared with CPF and CAL $(P<0.05$; Fig. 1(A) and (B)). Hepatic SFA C6:0, C12:0, C16:0 and C18:0 were enhanced in $\mathrm{RD}$ compared with CPF and CAL $(P<0 \cdot 05$; Fig. 2$)$. Total SFA

Table 1. Plasma parameters on day 21 of ducks in the riboflavin-deficient (RD), pair-fed with the control diet to the mean daily intake of the RD group (CPF) and ad libitum control (CAL) groups (n 12)*

\begin{tabular}{lccccr}
\hline Variables & RD & CPF & CAL & SEM & $P$ \\
\hline Glucose $(\mathrm{mmol} / \mathrm{l})$ & 7.48 & 8.07 & 7.48 & 0.13 & 0.111 \\
Lactate $(\mathrm{mmol} / \mathrm{l})$ & 5.57 & 6.14 & 6.37 & 0.24 & 0.408 \\
Uric acid $(\mu \mathrm{mol} / \mathrm{l})$ & $365^{\mathrm{a}}$ & $243^{\mathrm{b}}$ & $188^{\mathrm{b}}$ & 26.0 & 0.013 \\
Pyruvate $(\mathrm{mmol} / \mathrm{l})$ & $0.30^{\mathrm{a}}$ & $0.21^{\mathrm{b}}$ & $0.21^{\mathrm{b}}$ & 0.01 & $<0.001$ \\
Albumin $(\mathrm{g} / \mathrm{l})$ & $32.2^{\mathrm{a}}$ & $29.6^{\mathrm{b}}$ & $29.3^{\mathrm{b}}$ & 0.41 & 0.003 \\
TAG $(\mathrm{mmol} / \mathrm{l})$ & $1.05^{\mathrm{a}}$ & $0.87^{\mathrm{b}}$ & $0.88^{\mathrm{b}}$ & 0.03 & 0.008 \\
TC $(\mathrm{mmol} / \mathrm{l})$ & $7.97^{\mathrm{a}}$ & $7.18^{\mathrm{b}}$ & $6.91^{\mathrm{b}}$ & 0.17 & 0.026 \\
ALT $(\mathrm{U} / \mathrm{l})$ & $18.0^{\mathrm{a}}$ & $8.87^{\mathrm{b}}$ & $10.7^{\mathrm{a}, \mathrm{b}}$ & 0.93 & $<0.001$ \\
AST $(\mathrm{U} / \mathrm{l})$ & $24.5^{\mathrm{a}}$ & $8.33^{\mathrm{b}}$ & $9.00^{\mathrm{b}}$ & 2.13 & 0.001 \\
\hline
\end{tabular}

TC, total cholesterol; ALT, alanine transaminase; AST, aspartate transaminase.

a,b Mean values with unlike superscript letters were significantly different $(P<0.05)$.

* Data were analysed by one-way ANOVA followed by Tukey's multiple comparison.
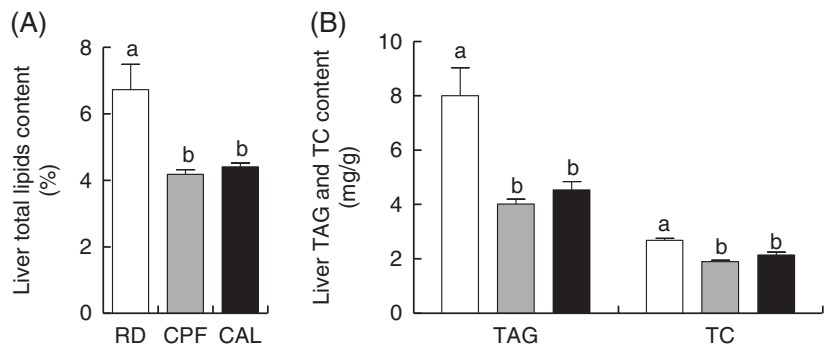

Fig. 1. Liver total lipid content (A), TAG and total cholesterol (TC) content (B) on day 21 of ducks in the riboflavin-deficient (RD, $\square$ ), pair-fed with the control diet to the mean daily intake of the RD group (CPF, $\square$ ) and ad libitum control (CAL, $\square$ ) groups, respectively. Values are means $(n 12)$, with their standard errors represented by vertical bars. ${ }^{a, b}$ Mean values with unlike letters were significantly different $(P<0.05)$. Data were analysed by one-way ANOVA followed by Tukey's multiple comparison.

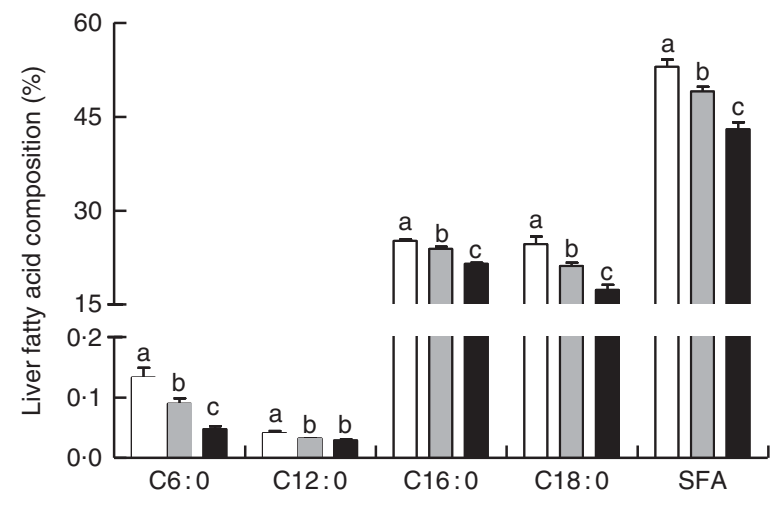

Fig. 2. Liver fatty acid composition (\% of the total fatty acid) on day 21 of ducks in the riboflavin-deficient (RD, $\square$ ), pair-fed with the control diet to the mean daily intake of the RD group (CPF, $\square$ ) and ad libitum control (CAL, $\square$ ) groups. Values are means $(n 12)$, with their standard errors represented by vertical bars. ${ }^{a, b, c}$ Mean values with unlike letters were significantly different $(P<0.05)$. Data were analysed by one-way ANOVA followed by Tukey's multiple comparison. 
content in liver was greater in $\mathrm{RD}$ than in $\mathrm{CPF}$ and $\mathrm{CAL}$ $(P<0.001$; Fig. 2). Complete liver fatty acid compositions are shown in the online Supplementary Table S3.

\section{Changes in the liver proteomics of duck from the three groups}

A total of 31851 peptide spectral matches were found, from which 1749 proteins were identified. Among the identified proteins, a total of sixty-three proteins showed an FC> 1.5 when comparing $\mathrm{RD}$ with $\mathrm{CAL}$ and $\mathrm{CPF}$, of which thirty-two were enhanced and thirty-one were diminished. The complete list of proteins regulated by RD is present in the online Supplementary Table S4. Of these, seven flavoproteins were regulated by RD, six diminished (ACADS, ACAD9, ACADM, hydroxyacid oxidase 2, dihydrolipoyl dehydrogenase (DLD) and electron transfer flavoprotein-ubiquinone oxidoreductase (ETFDH)) and one enhanced (xanthine dehydrogenase/oxidase).

Gene ontology enrichment analysis was performed for functional analysis of the sixty-three differentially expressed proteins using ClueGo software. The results showed that two major functional groups were enriched: lipid metabolism and cellular respiration (Fig. 3 and online Supplementary Table S5), and twenty-nine noteworthy proteins were involved in these processes (Table 2). The highest proportion of regulated proteins were those related to lipid metabolism, eight being diminished and fourteen enhanced. Fatty acid $\beta$-oxidation was mainly enriched in the diminished proteins (ACADS, ACADM, ACAD9, ETFDH), whereas the enhanced proteins were mainly enriched in TAG metabolic process (acyl-CoA thioesterase 1 (ACOT1), acyl-CoA synthetase family member 2 (ACSF2), longchain-fatty-acid-CoA ligase 1 (ACSL1), long-chain-fatty-acidCoA ligase 5 (ACSL5), complement component 3 (C3), solute carrier family 27, member 5 (SLC27A5), apo B-100 (APOB), microsomal TAG transfer protein (MTTP)) and cholesterol metabolic process (hydroxymethylglutaryl-CoA synthase (HMGCS1), acetyl-CoA acetyltransferase (ACAT2) and nonspecific lipid-transfer protein (SCP2)).

Of the proteins in the enriched cellular respiration category, six proteins were diminished, two involved in TCA cycle (DLD and malate dehydrogenase (MDH1)) and four involved in ETC (ACAD9, NADH-ubiquinone oxidoreductase $75 \mathrm{kDa}$ subunit
(NDUFS1), NADH dehydrogenase (ubiquinone) $1 \alpha$ subcomplex subunit 8 (NDUFA8) and frataxin (FXN)). One protein in this category was enhanced (solute carrier family 25 (aspartate/glutamate carrier), member 13).

A KEGG pathway enrichment analysis was performed on the sixty-three differentially expressed proteins. The top ten pathways including the number of proteins affected are listed in Table 3: valine, leucine and isoleucine degradation, propanoate metabolism, pyruvate metabolism, fatty acid degradation, butanoate metabolism, PPAR signalling pathway, synthesis and degradation of ketone bodies, glyoxylate and dicarboxylate metabolism, $\beta$-alanine metabolism and glycolysis/ gluconeogenesis.

\section{Western blot analysis}

To validate proteomic results, Western blot analyses of three differentially expressed proteins were conducted. Proteins involved in $\beta$-oxidation (ACADS and ACADM) and ETC (ACAD9) were selected. The Western blot results of ACADS, ACADM and ACAD9 were fully consistent with the findings from the iTRAQ analysis (Fig. 4).

\section{Discussion}

Previous studies described that RD could depress growth $^{(11-15,17-19,41)}$ and induce fatty livers ${ }^{(21,22)}$ of animals. In the present study, growth depression, liver enlargement and liver lipid accumulation were observed in RD ducks, accompanied by a high mortality rate. No overt pathology was found in the birds that died from RD. Most likely their death stems from the metabolic processes that were identified, which result in energy deficiency, a speculation that remains to be verified. Furthermore, poor riboflavin status was found in RD ducks indicated by a marked reduction of free riboflavin concentrations in liver and in plasma. These results demonstrate that a severe RD animal model was successfully established. Using, for the first time, a proteomic approach, the present study shows that RD mainly affects proteins involved in lipid metabolism and cellular respiration, suggesting these processes to probably underlie the liver lipid metabolic disorder and poor growth. Notably, almost all flavoproteins or subunits of

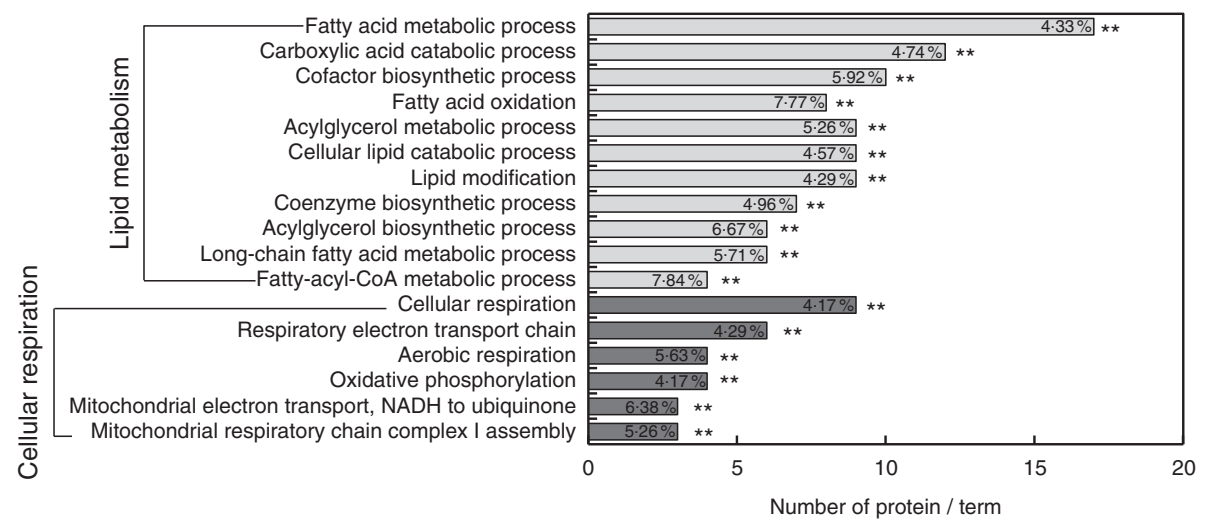

Fig. 3. Functional enrichment analysis of the differentially abundance proteins in liver regulated by riboflavin deficiency using ClueGO software. ${ }^{* \star}$ Significant enrichment at the $P<0.01$ statistical level. 
Table 2. Differentially expressed proteins in liver involved in lipid metabolism and cellular respiration on day 21 of ducks caused by riboflavin deficiency (RD)

\begin{tabular}{|c|c|c|c|c|c|}
\hline \multirow[b]{2}{*}{ Accession nos } & \multirow[b]{2}{*}{ Protein name } & \multirow[b]{2}{*}{ Gene name } & \multicolumn{3}{|c|}{ Fold change* } \\
\hline & & & RD v. CAL & RD v. CPF & CPF v. CAL \\
\hline \multicolumn{6}{|l|}{ Lipid metabolism } \\
\hline trlR7VXW2IR7VXW2_COLLI & Inositol polyphosphate 1-phosphatase & INPP1 & -83.3 & $-90 \cdot 9$ & 2.33 \\
\hline 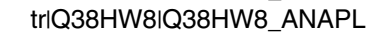 & Thyroid hormone responsive Spot $14 a$ & THRSP & -62.5 & -71.4 & 1.24 \\
\hline trIF1NAM7IF1NAM7_CHICK & 3-hydroxybutyrate dehydrogenase type 2 & $\mathrm{BDH} 2$ & $-23 \cdot 8$ & $-23 \cdot 3$ & 1.43 \\
\hline trlU3J1JOIU3J1J0_ANAPL & Acyl-CoA dehydrogenase family member 9 & ACAD9 & $-6 \cdot 41$ & -8.40 & 1.41 \\
\hline trlU3J8W0IU3J8W0_ANAPL & Short-chain-specific acyl-CoA dehydrogenase & ACADS & -4.74 & $-6 \cdot 80$ & 1.17 \\
\hline trlU3ITA9IU3ITA9_ANAAPL & Medium-chain-specific acyl-CoA dehydrogenase & ACADM & -2.99 & $-3 \cdot 25$ & 1.08 \\
\hline trlU3J3V1IU3J3V1__ANAPL & Electron transfer Flavoprotein-ubiquinone oxidoreductase & ETFDH & $-2 \cdot 88$ & $-3 \cdot 19$ & 1.06 \\
\hline tr|U3IQB4IU3IQB4_ANAPL & Hydroxyacid oxidase 2 & $\mathrm{HAO2}$ & -1.92 & $-1 \cdot 60$ & $-1 \cdot 21$ \\
\hline trlU3IJZ6IU3IJZ6_ĀNAPL & Fatty acid amide hydrolase & $F A A H$ & 1.59 & $2 \cdot 17$ & -1.36 \\
\hline trlU3J6POIU3J6PO_ANAPL & Complement component 3 & C3 & 1.79 & 6.86 & $-3 \cdot 83$ \\
\hline trlU3IKU2IU3IKU2_ANAPL & Non-specific lipid-transfer protein & $S C P 2$ & 1.92 & $2 \cdot 23$ & $-1 \cdot 13$ \\
\hline tr|U3IEH8IU3IEH8_ANAPL & Acetyl-CoA acetyltransferase & ACAT2 & 2.00 & 1.60 & 1.26 \\
\hline trlU3IWA4IU3IWA4_ANAPL & Apolipoprotein B-100 & $A P O B$ & 2.01 & 2.97 & -1.45 \\
\hline tr|ROK9VOIROK9V0_ANAPL & Acetyl-coA synthetase & ACS & $2 \cdot 31$ & 4.61 & $-2 \cdot 13$ \\
\hline tr|R0JGG2IR0JGG2_ANAPL & Hydroxymethylglutaryl-CoA synthase & HMGCS1 & 2.42 & 4.21 & $-1 \cdot 82$ \\
\hline tr|U3III1|U3|II1_ANAPL & Acyl-coA thioesterase 1 & ACOT1 & 2.61 & 4.17 & -1.66 \\
\hline trlU3I2B5IU3I2B5_ANAPL & Solute carrier family 27 (fatty acid transporter), member 5 & SLC27A5 & 2.75 & 5.45 & $-2 \cdot 29$ \\
\hline $\operatorname{tr}|U 3 J 3 G 1| U 3 J 3 G 1 \_A N A P L$ & Enoyl-CoA hydratase & ECHS1 & 2.83 & 2.78 & 1.05 \\
\hline tr|U3I559IU3I559_ANAPL & Microsomal TAG transfer protein & MTTP & 3.22 & $9 \cdot 20$ & -3.40 \\
\hline trIU3IU30IU3IU30 ANAPL & Long-chain-fatty-acid-CoA ligase 1 & ACSL1 & 3.37 & 4.17 & $-1 \cdot 20$ \\
\hline trlU3J4Z9IU3J4Z9_ANAPL & Long-chain-fatty-acid-CoA ligase 5 & ACSL5 & 3.57 & $9 \cdot 73$ & $-3 \cdot 25$ \\
\hline trlH0ZF88IH0ZF88_TAEGU & Acyl-CoA synthetase family member 2 & ACSF2 & $86 \cdot 3$ & $86 \cdot 3$ & -1.01 \\
\hline \multicolumn{6}{|l|}{ Cellular respiration } \\
\hline tr|R7VUZ3IR7VUZ3_COLLI & Frataxin & FXN & $-90 \cdot 9$ & -90.9 & -1.05 \\
\hline $\operatorname{tr|R0K082IR0K082~ANAPL~}$ & NADH-ubiquinone oxidoreductase $75 \mathrm{kDa}$ subunit & NDUFS1 & -11.4 & $-11 \cdot 1$ & $1 \cdot 15$ \\
\hline tr|U3IR48IU3IR48_ANAPL & Dihydrolipoyl dehydrogenase & $D L D$ & $-5 \cdot 71$ & $-8 \cdot 26$ & 1.47 \\
\hline tr|G1N071|G1N071_MELGA & Malate dehydrogenase & $\mathrm{MDH} 1$ & -1.98 & $-2 \cdot 27$ & $-1 \cdot 01$ \\
\hline 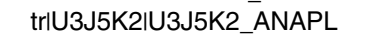 & NADH dehydrogenase (ubiquinone) $1 a$ subcomplex subunit 8 & NDUFA8 & -1.64 & -3.40 & 2.03 \\
\hline trIU3J8L3IU3J8L3_ANAPL & Solute carrier family 25 (aspartate/glutamate carrier), member 13 & SLC25A13 & 1.84 & 4.88 & $-2 \cdot 81$ \\
\hline $\operatorname{tr|U3|3S5|U3|3S5~ANAPL~}$ & Nicotinamide nucleotide transhydrogenase & NNT & $2 \cdot 21$ & 4.41 & $-2 \cdot 17$ \\
\hline
\end{tabular}

CPF, pair-fed with the CAL to the mean daily intake of the RD group; CAL, ad libitum control diet.

${ }^{*}$ Fold change is expressed as the ratio between two of three groups. For diminished proteins, the fold change was transformed to the corresponding negative value.

Table 3. Enriched Kyoto Encyclopedia of Genes and Genomes-pathway-based sets of differential levels of proteins in the liver of starter Pekin ducks in response to riboflavin deficiency*

\begin{tabular}{lccl}
\hline Pathway names & $P$ & Count & Protein \\
\hline Valine, leucine and isoleucine degradation & $2 \cdot 7 \mathrm{E}-10$ & 9 & ABAT, ACADM, ACADS, ACAT2, ALDH7A1, DBT, DLD, ECHS1, HMGCS1 \\
Propanoate metabolism & $3 \cdot 1 \mathrm{E}-10$ & 8 & ABAT, ACADM, ACAT2, ACS, DBT, DLD, ECHS1, LDHA \\
Pyruvate metabolism & $8 \cdot 6 \mathrm{E}-08$ & 7 & ACAT2, ACS, ALDH7A1, DLD, LDHA, MDH1, ME1 \\
Fatty acid degradation & $1 \cdot 6 \mathrm{E}-07$ & 7 & ACADM, ACADS, ACAT2, ACSL1, ACSL5, ALDH7A1, ECHS1 \\
Butanoate metabolism & $2 \cdot 6 \mathrm{E}-07$ & 6 & ABAT, ACADS, ACAT2, BDH2, ECHS1, HMGCS1 \\
PPAR signalling pathway & 0.000063 & 6 & ACADM, ACSL1, ACSL5, ME1, SCP2, SLC27A5 \\
Synthesis and degradation of ketone bodies & 0.00029 & 3 & ACAT2, BDH2, HMGCS1 \\
Glyoxylate and dicarboxylate metabolism & 0.00032 & 4 & ACAT2, DLD, HAO2, MDH1 \\
B-Alanine metabolism & 0.00039 & 4 & ABAT, ACADM, ALDH7A1, ECHS1 \\
Glycolysis/Gluconeogenesis & 0.00054 & 5 & ACS, ALDH7A1, DLD, LDHA, PFK
\end{tabular}

ABAT, 4-aminobutyrate aminotransferase; ACADM, medium-chain-specific acyl-CoA dehydrogenase; ACADS, short-chain-specific acyl-CoA dehydrogenase; ACAT2, acetyl-CoA acetyltransferase; ALDH7A1, aldehyde dehydrogenase 7 family, member A1; DBT, lipoamide acyltransferase component of branched-chain $a$-keto acid dehydrogenase complex; DLD, dihydrolipoyl dehydrogenase; ECHS1, enoyl-CoA hydratase; HMGCS1, hydroxymethylglutaryl-CoA synthase; ACS, acetyl-coA synthetase; LDHA, L-lactate dehydrogenase; $\mathrm{MDH} 1$, malate dehydrogenase; ME1, malic enzyme; ACSL1, long-chain-fatty-acid-CoA ligase 1; ACSL5, long-chain-fatty-acid-CoA ligase 5; BDH2, 3-hydroxybutyrate dehydrogenase type 2; SCP2, non-specific lipid-transfer protein; SLC27A5, solute carrier family 27 (fatty acid transporter), member 5; HAO2, hydroxyacid oxidase 2; PFK; 6-phosphofructokinase.

* The top ten pathways obtained are sorted based on significance.

flavin-dependent enzymes among the RD altered proteins were reduced in the liver from RD ducks, such as ACADS, ACADM, ACAD9, ETFDH and DLD. This finding is consistent with the hypothesis that flavoprotein expression may be down-regulated owing to reduced supply of riboflavin in the diet and is in line with a human study ${ }^{(42)}$.

Previous studies proved that RD markedly decreased feed intake in rats ${ }^{(11-15,41)}$, pigs ${ }^{(43)}$ and ducks ${ }^{(17-19)}$. As expected, an 
(A)
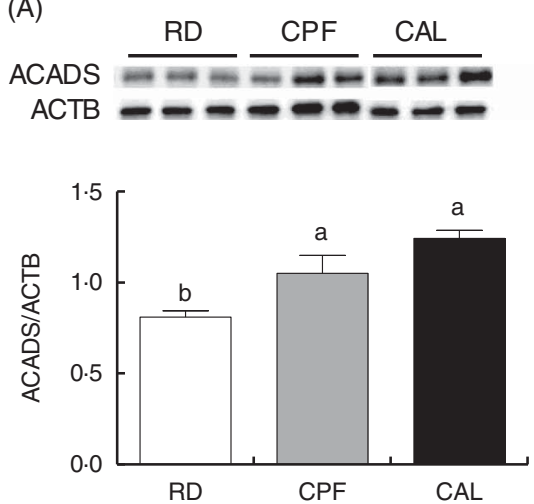

(B)
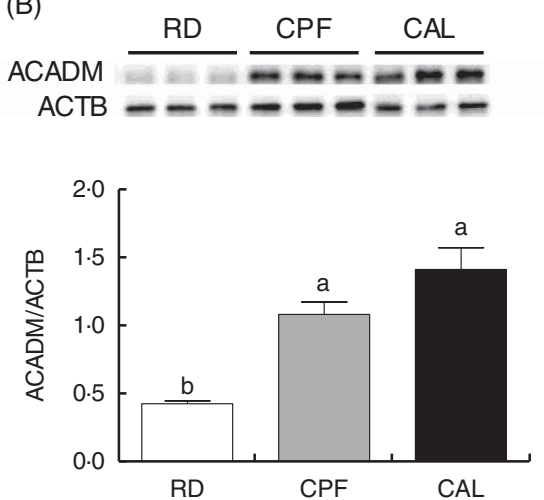

(C)
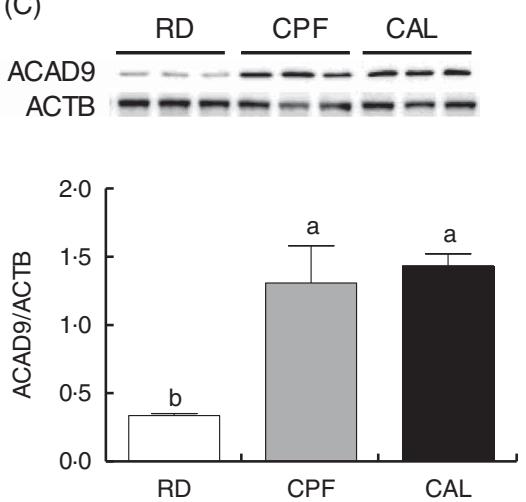

Fig. 4. Western blot analysis of short-chain-specific acyl-CoA dehydrogenase (ACADS; A) medium-chain-specific acyl-CoA dehydrogenase (ACADM; B) and acylCoA dehydrogenase family member 9 (ACAD9; C) protein abundance of liver tissue of ducks in the riboflavin-deficient (RD), pair-fed with the control diet to the mean daily intake of the RD group (CPF) and ad libitum control (CAL) groups. $\beta$-Actin (ACTB) was used as a loading control. Representative Western blots are shown. Values are means $(n 6)$, with their standard errors represented by vertical bars. ${ }^{a, b}$ Mean values with unlike letters were significantly different $(P<0.05)$. Data were analysed by one-way ANOVA followed by Tukey's multiple comparison.

$\mathrm{RD}$ diet in the present study led to a decreased overall feed intake. The inclusion of a CPF group, in addition to a CAL group, allowed to conclude that the biochemical or molecular changes observed in the $\mathrm{RD}$ group were attributable to $\mathrm{RD}$ and not to an unequal feed intake.

In the present study, RD caused liver damage indicated by elevated plasma ALT and AST activities, confirming our previous findings ${ }^{(18)}$. Additional evidence for liver damage was provided by the markedly increased relative liver weight in $\mathrm{RD}$ ducks, which is indicative of liver enlargement, confirming previous findings in other animal species ${ }^{(11-13,15,41,43-45)}$. Liver enlargement in the $\mathrm{RD}$ group might due to lipid accumulation indicated by elevation of liver total lipids, TAG and TC, which is consistent with previous findings ${ }^{(12,45,46)}$. Together, these results provide evidence that the liver responds sensitively to $\mathrm{RD}$, making it an interesting organ for extended proteomic analysis to investigate the mechanisms underlying growth depression and lipid accumulation.

\section{Lipid metabolism}

A total of twenty-two proteins participating in the lipid metabolism pathway were differentially expressed after $\mathrm{RD}$, making it the largest category of identified proteins. Of these, five proteins were involved in fatty acid $\beta$-oxidation, one enhanced (enoyl-CoA hydratase) and four diminished (ACADS, ACADM, ACAD9 and ETFDH; Fig. 5). ACADS, ACADM and ACAD9 belong to the family of fatty acyl-CoA dehydrogenases, which catalyse the initial rate-limiting step of the $\beta$-oxidation cycle ${ }^{(28)}$. ETFDH accepts electrons from multiple acetyl-CoA dehydrogenases and subsequently transfers these to $\operatorname{ETC}^{(47)}$. Therefore, a reduction of ACADS, ACADM, ACAD9 and ETFDH suggests that the rate of fatty acid $\beta$-oxidation in the liver may be impaired in RD ducks, which is supported by previous studies in rodents ${ }^{(12,15,24-27)}$. Furthermore, ACADS and ACADM catalyse oxidation of fatty acids of 4-6 carbons and 4-16 carbons, respectively ${ }^{(28)}$, whereas ACAD9 catalyses oxidation of palmitoyl-CoA $(\mathrm{C} 16: 0)$ and stearoyl-CoA $(\mathrm{C} 18: 0)^{(48)}$.

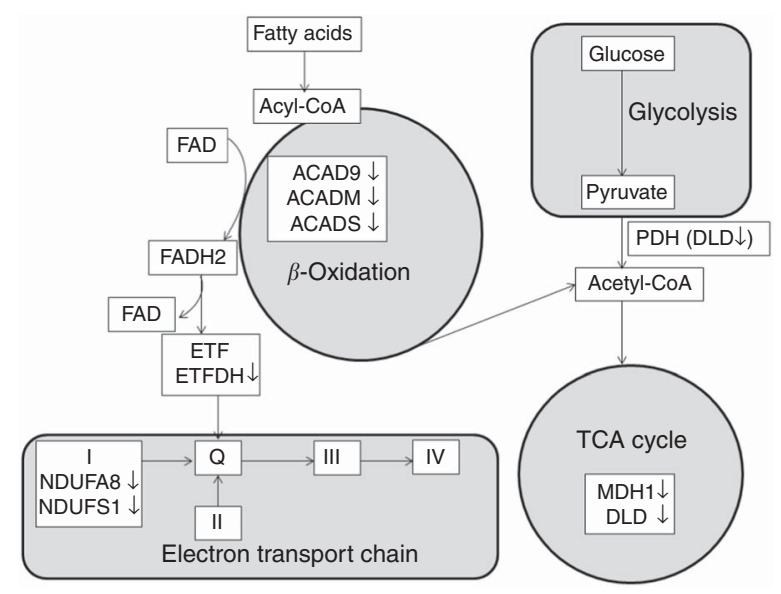

Fig. 5. Differentially expressed proteins involved in fatty acid $\beta$-oxidation, tricarboxylic acid (TCA) cycle and electron transport chain. Protein names for the symbols used are defined in Table 3. (on the right), diminished protein levels in response to riboflavin deficiency; FADH2, 1, 5-dihydro-FAD; ETF, electron transfer flavoprotein; ETFDH, electron transfer flavoprotein-ubiquinone oxidoreductase; ACAD9, acyl-CoA dehydrogenase family member 9; ACADM, medium-chain specific acyl-CoA dehydrogenase; ACADS, short-chain-specific acyl-CoA dehydrogenase; $\mathrm{PDH}$, pyruvate dehydrogenase complex; DLD, dihydrolipoyl dehydrogenase; MDH1, malate dehydrogenase; NDUFA8, $\mathrm{NADH}$ dehydrogenase (ubiquinone) 1a subcomplex subunit 8; NDUFS1 (Anapl_00655), NADH-ubiquinone oxidoreductase $75 \mathrm{kDa}$ subunit; I, respiratory chain complex I (NADH-ubiquinone oxidoreductase); II, respiratory chain complex II (succinate dehydrogenase); Q, ubiquinone; III, respiratory chain complex III (ubiquinol-cytochrome c reductase); IV, respiratory chain complex IV (cytochrome coxidase).

Decreased protein expression of ACADS, ACADM and ACAD9 due to RD may provide a possible explanation for the observed elevation of the SFA C6:0, C12:0, C16:0 and C18:0, which probably leads to the lipid accumulation that is seen. This explanation is supported by findings in a riboflavin-responsive multiple acyl-CoA dehydrogenase deficiency (RR-MAD) patient, showing that the reduction of ACADS, ACADM and ETFDH protein levels lead to lipid accumulation in skeletal muscle, suggested to be due to defective $\beta$-oxidation ${ }^{(42)}$. 
In the present study, nine proteins that were differentially affected by RD are involved in TAG metabolic processes. Of these, one protein was diminished (Thyroid hormone responsive Spot $14 \alpha$ ), whereas eight proteins were enhanced (ACOT1, ACSF2, ACSL1, ACSL5, C3, SLC27A5, APOB and MTTP). ACOT1 hydrolyses acyl-CoA of $\mathrm{C}_{12}-\mathrm{C}_{20}$-CoA into long-chain free fatty acids and $\mathrm{CoA}^{(49,50)}$, and regulates cellular pools and proper ratios of activated fatty acyl-CoA, free fatty acids and $\mathrm{COASH}^{(51)}$. ACSF2, ACSL1 and ACSL5 belong to the acyl-CoA synthetase family ${ }^{(52,53)}$, which plays an important part in de novo lipid synthesis and fatty acid catabolism, catalysing the initial fatty acid activation by forming a thioester with $\mathrm{COA}^{(52,54)}$. SLC27A5 activates long-chain fatty acids but also enhances long-chain fatty acid uptake in the liver ${ }^{(55)}$. Together, the increase of ACOT1, ACSF2, ACSL1, ACSL5 and SLC27A5 in response to RD likely indicates increased activation, uptake of hepatic (longchain) fatty acids and probably subsequent enhanced lipid biosynthesis. These results might explain the increased TAG content observed in the liver of the RD group. In addition, APOB and MTTP play a critical role in the mobilisation and secretion of TAG-rich lipoproteins from hepatocytes ${ }^{(56)}$. APOB is required for formation of chylomicrons, VLDL and LDL and play a vital role in plasma lipoprotein metabolism ${ }^{(57)}$. MTTP is involved in lipid transfer during the assembly of lipoproteins in the liver and intestine ${ }^{(58)}$. Therefore, overexpression of MTTP and $\mathrm{APOB}$ in the RD group might explain the enhanced plasma TAG concentration observed in RD ducks.

Proteins involved in cholesterol metabolic processes were also affected by RD in the present study via three enhanced proteins: HMGCS1, ACAT2 and SCP2. HMGCS1 catalyses the first committed step in the mevalonate pathway, condensing acetoacety-CoA and acetyl-CoA to form 3-hydroxy-3-methylglutaryl-CoA ${ }^{(59)}$, which is an intermediate in cholesterol biosynthesis. The increased levels of HMGCS1 in RD might suggest enhancement of cholesterol synthesis, providing a possible explanation for the enhanced TC concentration in liver or plasma in response to RD. Similarly, the enhanced levels of ACAT2 and SCP2 might contribute to the explanation of increased TC levels in RD ducks. SCP2 plays an important role in cholesterol transport, cholesterol esterification and bile acid formation $^{(60)}$. ACAT2 is an enzyme responsible for intracellular cholesterol esterification, and inhibition of ACAT2 activity in the liver decreases serum cholesterol levels ${ }^{(61)}$. The overexpression of HMGCS1, ACAT2 and SCP2 in liver provides a possible explanation for the enhanced TC concentrations in liver or plasma in response to RD.

\section{Cellular respiration}

RD diminished two key enzymes involved in TCA cycle (DLD and MDH1; Fig. 5) and four proteins involved in ETC function (ACAD9, NDUFS1, NDUFA8 and FXN; Fig. 5). Results obtained in a previous human study, showing that NDUFS1, NDUFA8 and DLD were diminished in skeletal muscle of RR-MAD patient ${ }^{(42)}$ validate our results. DLD (E3) is the common component of pyruvate and $\alpha$-ketoglutarate dehydrogenase complexes, converting dihydrolipoic acid and $\mathrm{NAD}^{+}$into lipoic acid and $\mathrm{NADH}^{(62)}$. Pyruvate dehydrogenase catalyses the oxidative decarboxylation of pyruvate to acetyl-CoA, thereby linking glycolysis to the TCA cycle and fatty acid synthesis ${ }^{(63,64)}$. The decreased expression of DLD in the RD group might lead to the accumulation of plasma pyruvate. $\alpha$-Ketoglutarate dehydrogenase is a rate-limiting enzyme of the TCA cycle, catalysing the conversion of $\alpha$-ketoglutarate to succinyl-CoA and $\mathrm{NADH}^{(65,66)}$. MDH1 catalyses the reversible interconversion of malate and oxaloacetate. Therefore, the reduced expression of DLD and MDH1 in the RD group probably indicates a decreased liver TCA cycle.

$\mathrm{RD}$ diminished proteins involved in the ETC, including ACAD9, NDUFS1 and NDUFA8, and FXN. ACAD9 not only plays a physiological role in fatty acid oxidation, but is also an assembly factor for mitochondrial respiratory chain complex $I^{(67)}$. As two subunits of complex I, NDUFS1 and NDUFA8 play a direct role in complex I assembly ${ }^{(68,69)}$. The decrease of these proteins in $\mathrm{RD}$ is in line with the role of riboflavin in complex I assembly ${ }^{(70)}$. This suggestion is supported by previous findings that either ACAD9 or NDUFS1 defects or mutations cause complex I deficiency ${ }^{(71-75)}$. FXN is not only involved in iron-sulphur cluster assembly ${ }^{(76)}$ but is also able to physically interact with complex II and the electron transfer flavoprotein complex ${ }^{(77)}$. The diminished expression of FXN in RD is supported by data showing that FXN deficiency can be rescued by FAD supplementation, enhancing mitochondrial respiration ${ }^{(78)}$. This finding suggests that RD probably block the ETC process not only via complex I but also via complex II. Indeed, it has been reported in a previous study that there was loss of activity of the flavin-dependent sections in complex I and II of the ETC due to RD ${ }^{(15)}$. Decreased expression of proteins involved in the ETC process likely imply that mitochondrial oxidative phosphorylation is impaired in response to $\mathrm{RD}$. The previous observation that oxygen consumption and oxidative phosphorylation in liver mitochondria of RD rodents were significantly lower than those in the control group supports this implication ${ }^{(27,79,80)}$.

Our proteomic analysis revealed that fatty acid $\beta$-oxidation, TCA cycle and oxidative phosphorylation processes were probably impaired in RD ducks, which may lead to insufficient ATP production and subsequent growth retardation. This result might explain reduced energy utilisation for growth owing to $\mathrm{RD}$ indicated by markedly reduced weight gain per unit of feed consumed in this study, which is also observed in previous studies in rats ${ }^{(12-15,41,81)}$ and pigs ${ }^{(43)}$. The results support the hypothesis that the lower food utilisation efficiency as the consequence of $\mathrm{RD}$ is due to incomplete oxidation of nutrients ${ }^{(80,82,83)}$

\section{Conclusion}

Proteomics analysis in liver of RD ducks showed that RD mainly affected proteins involved in lipid metabolism and cellular respiration. Down-regulation of flavoproteins involved in fatty acid $\beta$-oxidation (ACADS, ACADM, ACAD9 and ETFDH) probably indicate impaired $\beta$-oxidation of fatty acids, which may lead to liver lipid accumulation. Furthermore, reduced expression of proteins involved in cellular respiration (DLD, MDH1, ACAD9, NDUFS1, NDUFA8 and FXN) may impair oxidative 
phosphorylation and thus reduce energy generation, which might lead to growth depression. This finding adds to our understanding of the mechanisms underlying lipid metabolic disorders as a result of RD.

\section{Acknowledgements}

This work was supported by the earmarked fund for China Agriculture Research System (CARS-42), the science and technology innovation project of Chinese Academy of Agricultural Sciences (CXGC-IAS-09) and the Chinese Scholarship Council (grant nos 201403250064 for J. T. and 201303250054 for W. S.).

J. T. performed the study, was involved in all aspects of analysis and drafted the manuscript. J. H., Y. G., M. X. and S. H. were involved in experimental design. M. X. performed data analysis, and J. H. and Y. J. performed sample analysis. J. T., M. A. H., W. S., V. d. B. and S. H. and J. K. were involved in the data interpretation and writing. All authors revised and approved the final version of the paper and agreed to be accountable for all aspects of the work in ensuring that questions related to the accuracy or integrity of any part of the work are appropriately investigated and resolved. All persons designated as authors qualify for authorship, and all those who qualify for authorship are listed.

The authors declare that there are no conflicts of interest.

\section{Supplementary material}

For supplementary material/s referred to in this article, please visit https://doi.org/10.1017/S0007114517002641

\section{References}

1. Powers HJ (2003) Riboflavin (vitamin B-2) and health. Am J Clin Nutr 77, 1352-1360.

2. Rohner F, Zimmermann MB, Wegmueller R, et al. (2007) Mild riboflavin deficiency is highly prevalent in school-age children but does not increase risk for anaemia in Cote d'Ivoire. $\mathrm{Br} \mathrm{J}$ Nutr 97, 970-976.

3. Boisvert WA, Castañeda C, Mendoza I, et al. (1993) Prevalence of riboflavin deficiency among Guatemalan elderly people and its relationship to milk intake. Am J Clin Nutr 58, 85-90.

4. Powers HJ, Hill MH, Mushtaq S, et al. (2011) Correcting a marginal riboflavin deficiency improves hematologic status in young women in the United Kingdom (RIBOFEM). Am J Clin Nutr 93, 1274-1284.

5. Whitfield KC, Karakochuk CD, Liu Y, et al. (2015) Poor thiamin and riboflavin status is common among women of childbearing age in rural and urban Cambodia. J Nutr 145, 628-633.

6. Neumann CG, Bwibo NO, Murphy SP, et al. (2003) Animal source foods improve dietary quality, micronutrient status, growth and cognitive function in Kenyan school children: background, study design and baseline findings. J Nutr $\mathbf{1 3 3}$, 3941S-3949S.

7. Lienhart W-D, Gudipati V \& Macheroux P (2013) The human flavoproteome. Arch Biochem Biophys 535, 150-162.

8. Venkataswamy G (1967) Efficacy of neera in curing riboflavin deficiency diseases. Indian J Nutr Diet 4, 271-276.

9. Batey DW, Daneshgar KK \& Eckhert CD (1992) Flavin levels in the rat retina. Exp Eye Res 54, 605-609.
10. Lane M \& Alfrey CP (1965) The anemia of human riboflavin deficiency. Blood 25, 432-442.

11. Kim YS \& Lambooy JP (1969) Biochemical and physiological changes in the rat during riboflavin deprivation and supplementation. J Nutr 98, 467-476.

12. Olpin S \& Bates C (1982) Lipid metabolism in riboflavindeficient rats. Br J Nutr $\mathbf{4 7}, 577-588$.

13. Patterson B \& Bates C (1989) Riboflavin deficiency, metabolic rate and brown adipose tissue function in sucking and weanling rats. Br J Nutr 61, 475-483.

14. Duerden JM \& Bates C (1985) Effect of riboflavin deficiency on reproductive performance and on biochemical indices of riboflavin status in the rat. Br J Nutr 53, 97-105.

15. Veitch K, Draye J-P, Vamecq J, et al. (1989) Altered acyl-CoA metabolism in riboflavin deficiency. Biochim Biophys Acta 1006, 335-343.

16. Deng D-F \& Wilson RP (2003) Dietary riboflavin requirement of juvenile sunshine bass (Morone chrysops $9 \times$ Morone saxatilis $\left.{ }^{+}\right)$. Aquaculture 218, 695-701.

17. Tang J, Xie M, Yang J, et al. (2013) Riboflavin requirements of white Pekin ducks from hatch to 21 d of age. Br Poult Sci $\mathbf{5 4}$, 407-411.

18. Tang J, Wen ZG, Guo ZB, et al. (2014) Dietary riboflavin supplementation improve the growth performance and antioxidant status of starter white Pekin ducks fed a corn-soybean meal diets. Livest Sci 170, 131-136.

19. Tang J, Hu J, Wen ZG, et al. (2015) Effects of riboflavin supplementation on growth performance, carcass traits, and riboflavin status of growing male white Pekin ducks. Anim Feed Sci Technol 209, 274-279.

20. Gunanti IR, Marks GC, Al-Mamun A, et al. (2014) Low serum vitamin B-12 and folate concentrations and low thiamin and riboflavin intakes are inversely associated with greater adiposity in Mexican American children. J Nutr 144, 2027-2033.

21. Gershoff S, Andrus S \& Hegsted D (1959) The effect of the carbohydrate and fat content of the diet upon the riboflavin requirement of the cat. $J$ Nutr $\mathbf{6 8}, 75-88$.

22. Taniguchi M \& Nakamura M (1976) Effects of riboflavin deficiency on the lipids of rat liver. J Nutr Sci Vitaminol 22, 135-146.

23. Taniguchi M, Yamamoto T \& Nakamura M (1978) Effects of riboflavin deficiency on the lipids of rat liver mitochondria and microsomes. J Nutr Sci Vitaminol 24, 363-381.

24. Hoppel C, DiMarco JP \& Tandler B (1979) Riboflavin and rat hepatic cell structure and function. Mitochondrial oxidative metabolism in deficiency states. $J$ Biol Chem 254, 4164-4170.

25. Sakurai T, Miyazawa S, Furuta S, et al. (1982) Riboflavin deficiency and $\beta$-oxidation systems in rat liver. Lipids $\mathbf{1 7}$, 598-604.

26. White HB III (1996) Sudden death of chicken embryos with hereditary riboflavin deficiency. J Nutr 126, 1303S-1317S.

27. Brijlal S, Lakshmi A \& Bamji M (1999) Mitochondrial oxidative metabolism during respiratory infection in riboflavin deficient mice. J Nutr Biochem 10, 728-732.

28. Ghisla S \& Thorpe C (2004) Acyl-CoA dehydrogenases. Eur J Biochem 271, 494-508.

29. National Research Council (1994) Nutrient Requirements of Poultry, 9th ed. Washington, DC: National Academies Press.

30. Britton NL, Riter KL, Smallidge RL, et al. (2003) Reversedphase liquid chromatographic determination of riboflavin in feeds. J AOAC Int 86, 197-201.

31. Batey DW \& Eckhert CD (1990) Identification of FAD, FMN, and riboflavin in the retina by microextraction and highperformance liquid chromatography. Anal Biochem 188, $164-167$. 
32. Petteys BJ \& Frank EL (2011) Rapid determination of vitamin $\mathrm{B}_{2}$ (riboflavin) in plasma by HPLC. Clin Chim Acta 412, 38-43.

33. Folch J, Lees M \& Sloane Stanley GH (1957) A simple method for the isolation and purification of total lipides from animal tissues. J Biol Chem 226, 497-509.

34. Wen Z, Tang J, Hou S, et al. (2014) Choline requirements of White Pekin ducks from hatch to 21 days of age. Poult Sci $\mathbf{9 3}$, 1-6.

35. Shantha N \& Ackman R (1990) Nervonic acid versus tricosanoic acid as internal standards in quantitative gas chromatographic analyses of fish oil longer-chain $n-3$ polyunsaturated fatty acid methyl esters. J Chromatogr $\mathbf{5 3 3}$, $1-10$.

36. Yin J, Shang X, Li D, et al. (2008) Effects of dietary conjugated linoleic acid on the fatty acid profile and cholesterol content of egg yolks from different breeds of layers. Poult Sci $\mathbf{8 7}$, 284-290.

37. Wang C, Zheng A, Xie M, et al. (2014) Hypothalamic protein profiles associated with inhibited feed intake of ducks fed with insufficient dietary arginine. Animal $\mathbf{8}$, 1113-1118.

38. Bindea G, Mlecnik B, Hackl H, et al. (2009) ClueGO: a Cytoscape plug-in to decipher functionally grouped gene ontology and pathway annotation networks. Bioinformatics 25, 1091-1093.

39. Ashburner M, Ball CA, Blake JA, et al. (2000) Gene Ontology: tool for the unification of biology. Nat Genet $\mathbf{2 5}$, 25-29.

40. Zhu Y-W, Lu L, Li W-X, et al. (2015) Effect of dietary manganese on antioxidant status and expression levels of heat-shock proteins and factors in tissues of laying broiler breeders under normal and high environmental temperatures. Br J Nutr 114, 1965-1974.

41. Brady PS, Feng Y-X \& Brady LJ (1988) Transcriptional regulation of carnitine palmitoyltransferase synthesis in riboflavin deficiency in rats. J Nutr 118, 1128-1136.

42. Gianazza E, Vergani L, Wait R, et al. (2006) Coordinated and reversible reduction of enzymes involved in terminal oxidative metabolism in skeletal muscle mitochondria from a riboflavinresponsive, multiple acyl-CoA dehydrogenase deficiency patient. Electrophoresis 27, 1182-1198.

43. Brady PS, Brady LJ, Parsons MJ, et al. (1979) Effects of riboflavin deficiency on growth and glutathione peroxidase system enzymes in the baby pig. J Nutr 109, 1615-1622.

44. Guggenheim K \& Diamant E (1959) Body composition of rats in B-vitamin deficiencies. Br J Nutr 13, 61-67.

45. Duerden JM \& Bates C (1985) Effect of riboflavin deficiency on lipid metabolism of liver and brown adipose tissue of sucking rat pups. Br J Nutr 53, 107-115.

46. Liao F \& Huang P (1987) Effects of moderate riboflavin deficiency on lipid metabolism in rats. Proc Natl Sci Counc Repub China B 11, 128-132.

47. Olsen RK, Andresen BS, Christensen E, et al. (2003) Clear relationship between ETF/ETFDH genotype and phenotype in patients with multiple acyl-CoA dehydrogenation deficiency. Hum Mutat 22, 12-23.

48. Zhang J, Zhang W, Zou D, et al. (2002) Cloning and functional characterization of ACAD-9, a novel member of human acylCoA dehydrogenase family. Biochem Biophys Res Commun 297, 1033-1042.

49. Dongol B, Shah Y, Kim I, et al. (2007) The acyl-CoA thioesterase I (Acot1) is regulated by the peroxisome proliferatoractivated receptor alpha and hepatocyte nuclear factor 4 alpha via a distal response element in the promotor. J Lipid Res $\mathbf{4 8}$ $1781-1791$
50. Hunt MC, Yamada J, Maltais LJ, et al. (2005) A revised nomenclature for mammalian acyl-CoA thioesterases/hydrolases. J Lipid Res 46, 2029-2032.

51. Brocker C, Carpenter C, Nebert DW, et al. (2010) Evolutionary divergence and functions of the human acyl-CoA thioesterase gene (ACOT) family. Hum Genomics $\mathbf{4}$, 411-420.

52. Watkins PA, Maiguel D, Jia Z, et al. (2007) Evidence for 26 distinct acyl-coenzyme A synthetase genes in the human genome. J Lipid Res 48, 2736-2750.

53. Yu S, Wei W, Xia M, et al. (2016) Molecular characterization, alternative splicing and expression analysis of ACSF2 and its correlation with egg-laying performance in geese. Anim Genet 47, 451-462.

54. Soupene E \& Kuypers FA (2008) Mammalian long-chain acylCoA synthetases. Exp Biol Med 233, 507-521.

55. Doege H, Baillie RA, Ortegon AM, et al. (2006) Targeted deletion of FATP5 reveals multiple functions in liver metabolism: alterations in hepatic lipid homeostasis. Gastroenterology 130, 1245-1258.

56. Xie Y, Newberry EP, Young SG, et al. (2006) Compensatory increase in hepatic lipogenesis in mice with conditional intestine-specific Mttp deficiency. J Biol Chem 281, 4075-4086.

57. Walldius G \& Jungner I (2006) The apoB/apoA-I ratio: a strong, new risk factor for cardiovascular disease and a target for lipid-lowering therapy-a review of the evidence. J Intern Med 259, 493-519.

58. Hussain MM, Shi J \& Dreizen P (2003) Microsomal triglyceride transfer protein and its role in apoB-lipoprotein assembly. J Lipid Res 44, 22-32.

59. Shafqat N, Turnbull A, Zschocke J, et al. (2010) Crystal structures of human HMG-CoA synthase isoforms provide insights into inherited ketogenesis disorders and inhibitor design. J Mol Biol 398, 497-506.

60. Weber F, Dyer J, Garcia FL, et al. (1998) In pre-sterol carrier protein 2 (SCP2) in solution the leader peptide 1-20 is flexibly disordered, and residues 21-143 adopt the same globular fold as in mature SCP2. Cell Mol Life Sci 54, 751-759.

61. Bell TA, Brown JM, Graham MJ, et al. (2006) Liver-Specific inhibition of acyl-coenzyme A: cholesterol acyltransferase 2 with antisense oligonucleotides limits atherosclerosis development in apolipoprotein B100-only low-density lipoprotein receptor-/- mice. Arterioscler Thromb Vasc Biol 26, 1814-1820.

62. Carothers DJ, Pons G \& Patel MS (1989) Dihydrolipoamide dehydrogenase: functional similarities and divergent evolution of the pyridine nucleotide-disulfide oxidoreductases. Arch Biochem Biophys 268, 409-425.

63. Sugden MC \& Holness MJ (2003) Recent advances in mechanisms regulating glucose oxidation at the level of the pyruvate dehydrogenase complex by PDKs. Am J Physiol Endocrinol Metab 284, E855-E862.

64. Holness M \& Sugden M (2003) Regulation of pyruvate dehydrogenase complex activity by reversible phosphorylation. Biochem Soc Trans 31, 1143-1151.

65. Gibson GE, Blass JP, Beal MF, et al. (2005) The $\alpha$-ketoglutarate-dehydrogenase complex. Mol Neurobiol 31, 43-63.

66. Zündorf G, Kahlert S, Bunik V, et al. (2009) $\alpha$-Ketoglutarate dehydrogenase contributes to production of reactive oxygen species in glutamate-stimulated hippocampal neurons in situ. Neuroscience 158, 610-616.

67. Schiff M, Haberberger B, Xia C, et al. (2015) Complex I assembly function and fatty acid oxidation enzyme activity of ACAD9 both contribute to disease severity in ACAD9 deficiency. Hum Mol Genet 24, 3238-3247. 
68. Lazarou M, McKenzie M, Ohtake A, et al. (2007) Analysis of the assembly profiles for mitochondrial-and nuclear-DNAencoded subunits into complex I. Mol Cell Biol 27, 4228-4237.

69. Szklarczyk R, Wanschers BF, Nabuurs SB, et al. (2011) NDUFB7 and NDUFA8 are located at the intermembrane surface of complex I. FEBS Lett $\mathbf{5 8 5}, \mathbf{7 3 7 - 7 4 3}$

70. Grad LI \& Lemire BD (2006) Riboflavin enhances the assembly of mitochondrial cytochrome $\mathrm{c}$ oxidase in C. elegans NADH-ubiquinone oxidoreductase mutants. Biochim Biophys Acta 1757, 115-122.

71. Gerards M, van den Bosch BJ, Danhauser K, et al. (2011) Riboflavin-responsive oxidative phosphorylation complex I deficiency caused by defective ACAD9: new function for an old gene. Brain 134, 210-219.

72. Haack TB, Danhauser K, Haberberger B, et al. (2010) Exome sequencing identifies ACAD9 mutations as a cause of complex I deficiency. Nat Genet 42, 1131-1134.

73. Nouws J, Nijtmans L, Houten SM, et al. (2010) Acyl-CoA dehydrogenase 9 is required for the biogenesis of oxidative phosphorylation complex I. Cell Metab 12, 283-294.

74. Martín MA, Blázquez A, Gutierrez-Solana LG, et al. (2005) Leigh syndrome associated with mitochondrial complex I deficiency due to a novel mutation in the NDUFS1 gene. Arch Neurol 62, 659-661.

75. Bénit P, Chretien D, Kadhom N, et al. (2001) Large-scale deletion and point mutations of the nuclear NDUFV1 and
NDUFS1 genes in mitochondrial complex I deficiency. Am J Hum Genet 68, 1344-1352.

76. Yoon T \& Cowan JA (2003) Characterization of frataxin as an iron donor for assembly of [2Fe-2S] clusters in ISU-type proteins. J Am Chem Soc 125, 6078-6084.

77. González-Cabo P, Vázquez-Manrique RP, García-Gimeno MA, et al. (2005) Frataxin interacts functionally with mitochondrial electron transport chain proteins. Hum Mol Genet 14, 2091-2098.

78. Gonzalez-Cabo P, Ros S \& Palau F (2010) Flavin adenine dinucleotide rescues the phenotype of frataxin deficiency. PLOS ONE 5, e8872.

79. Kielley WW \& Bronk JR (1957) Oxidative phosphorylation in sonic extracts of rat liver mitochondria. Biochim Biophys Acta 23, 448-449.

80. Burch HB, HUNTER FE Jr, Combs AM, et al. (1960) Oxidative enzymes and phosphorylation in hepatic mitochondria from riboflavin-deficient rats. J Biol Chem $\mathbf{2 3 5}$, 1540-1544.

81. Burch HB, Lowry OH, Padilla AM, et al. (1956) Effects of riboflavin deficiency and realimentation on flavin enzymes of tissues. J Biol Chem 223, 29-45.

82. Sure B (1941) Further observations on riboflavin as a food factor in economy of food utilization. $J$ Nutr $\mathbf{2 2}$, 295-301.

83. Sure B, Dichek M \& Citron MM (1941) Riboflavin as a factor in economy of food utilization. J Nutr 21, 453-460. 